\title{
An image dehazing method based on scene segmentation
}

\author{
Ebtesam Mohameed Alharbi ${ }^{1,2, a}$, Yilin Shan ${ }^{2, b}$, Peng Ge ${ }^{\star 1,2,3, c}$, Hong Wang $^{1,2, d}$ \\ ${ }^{1}$ Engineering Research Center for Optoelectronics of Guangdong Province, School of Electronics \\ and Information,South China University of Technology,Guangzhou510640,China; \\ ${ }^{2}$ School of Physics and Optoelectronics, South China University of Technology, Guangzhou 510640, \\ China; \\ ${ }^{3}$ Institute of Electronic and Information, Hangzhou Dianzi University, Hangzhou,310018, China.

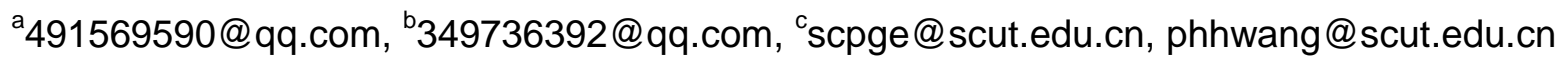 \\ Corresponding Author: Peng Ge
}

Keywords: Image processing, Image reconstruction-restoration, Dehazing.

\begin{abstract}
We propose an image dehazing method based on scene segmentation. We divide the hazy image into different parts depending on the scene depth. Then we dehaze the scenes in the same depth .Experimental results show that our method can achieve high color fidelity results. What's more, our results take advantage in plentiful details and much clearer in the far field's scene.
\end{abstract}

\section{Introduction}

Imaging devices are often affected by the particles in the atmosphere in foggy weather. With the influence of the atmosphere the quality of the image will degrade greatly and the outdoor visual system can't work normally in this situation.

At present the main dehazing method of single frame can be cataloged into two aspects. One is based on image enhancement. This algorithm doesn't consider the physical model of imaging principle. It only improves the image quality to satisfy our need by enhancing the contrast of the images and highlighting the image detail, such as histogram equalization[1], retinex theory [2], and so on. The other dehazing algorithm is based on image recovery [3-11]. This kind of algorithm is based on the physical model of atmosphere scattering. It recovers the image by some auxiliary information or some prior law.

Hazy images' contrast is related to the scene depth, the deeper the depth is, the brighter the pixel. It is easy to dehaze image if the whole image is in the same scene depth, but hazy image is usually in different scene depth. In this paper, we will segment the image scene depending on depth and process different parts in the same scene depth.

\section{The relationship between the hazy image and the scene depth}

The atmosphere scattering model refers to the total radiation that imaging devices receive including the radiation of the attenuated incident light [12] and the radiation of the atmosphere imaging system [13], that is

$$
I(x)=I_{\infty} \rho e^{-\beta d}+I_{\infty}\left(1-e^{-\beta d}\right),
$$

in which $I_{\infty}$ means the intensity of the environmental light, $\rho$ is the reflectance of the scene. $\beta$ is the scattering coefficient. $d$ means the depth of field. $I_{\infty} \rho$ means the radiation of the scene. $e^{-\beta d}$ means the transitivity of the scene. It shows the un-attenuated portion of the incident light which passes through the atmosphere and reaches to the imaging devices. As the depth of the field $\mathrm{d}$ increases, $e^{-\beta d}$ becomes smaller. When $e^{-\beta d}$ is smaller, it indicates that the attenuated portion is much more. $I_{\infty} \rho e^{-\beta d}$ means the attenuated portion of the incident light. $I_{\infty}\left(1-e^{-\beta d}\right)$ shows the effect that the 
environmental illumination brings to the imaging processing. We can see that when the depth of the field increases, the scene radiation attenuates much more, and it becomes less in the imaging processing, but the portion of the environmental radiation in the imaging processing becomes much larger. It results in the low contrast of the far scene in the image and the image shows wholly white. Here, we assume component $I_{\infty}$ and $\beta$ are the same for R,G,B channel, so Eq.(1) can be transfer to:

$$
\begin{aligned}
& I(x)=I_{\infty}\left(1-\mathrm{e}^{-\beta d}(1-R)\right) \\
& I(x)=I_{\infty}\left(1-\mathrm{e}^{-\beta d}(1-G)\right) \\
& I(x)=I_{\infty}\left(1-\mathrm{e}^{-\beta d}(1-B)\right) .
\end{aligned}
$$

Then we take the minimum operation to the three color channels in Eq.(2) and get:

$$
I(x)=I_{\infty}\left(1-\mathrm{e}^{-\beta d}(1-\min (R, G, B))\right) \text {. }
$$

Generally speaking, images have higher saturation in the good weather condition, and the value of item $\min (R, G, B)$ is small. So after processing by Gauss filtering, the value of item min(R,G,B) will be approximately equal in a local area. So the pixel value of $\min (R, G, B)$ image is mainly related to the scene depth. That is to say two different pixels in the same scene depth will have approximately the equal value. So we can segment hazy image depending on the pixel value of min(R,G,B) image.

\section{3.dehazing method based on image segmentation}

We first calculate min(R,G,B) item of the hazy image, and process the min(R,G,B) image by a Gauss filtering. According to our theory in the upper chapter, we can select several intervals of pixel value, and segment image depend on these intervals' range. Figure 1 shows the results:

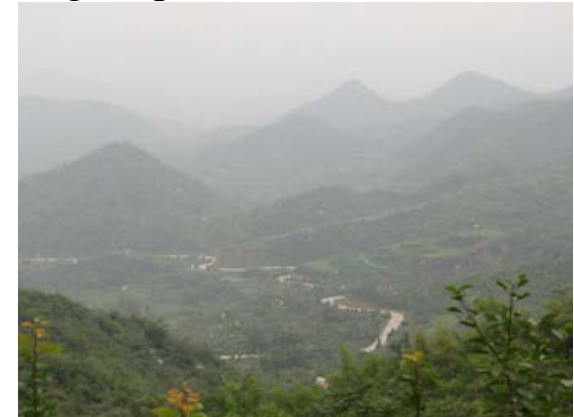

(a)

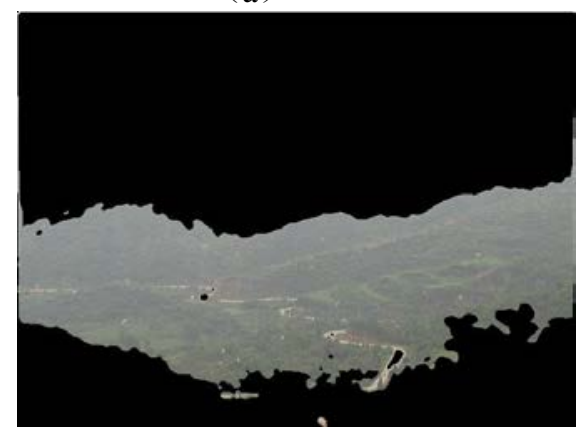

(c)

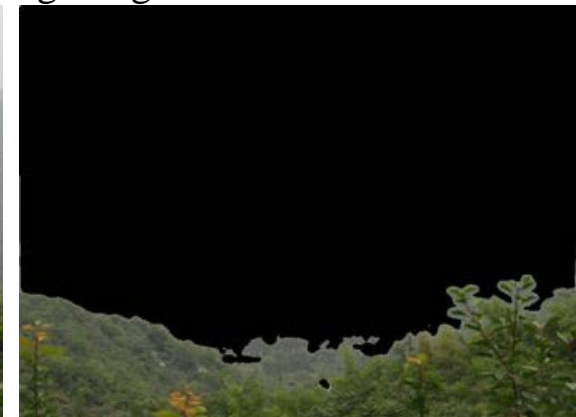

(b)

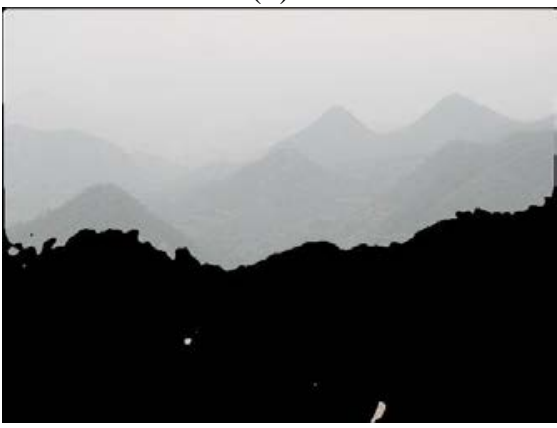

(d)

Figure 1 (a)Original image (b) min(R,G,B) image's pixel value interval $(0,100)$

(c) $\min (R, G, B)$ image's pixel value interval $(100,150)$; (d) $\min (R, G, B)$ image's pixel value interval $(150,255)$

From the results we can see that, original image was divided into three parts depending on the intervals' range. After segmentation each field can be seen as in the same scene depth. So we can assume the scene depth $d$ is almost invariant in the each field. From Eq. (3) we can get that the order of pixel value is only decided by the item $\rho$. So we can just use gray stretch method to process each field, it can ignore the influence of hazy and enhance the contrast of item $\rho$. Then we will get a dehazing image. 
Here we use the method of local overlapped to process the gray stretch. But we don't select subinterval in the hazy image space. Instead we select subinterval in $\min (R, G, B)$ image, and process each interval by the gray stretch. Specific steps as follows:

1. Calculate item min(R,G,B) from hazy image;

2. Use Gaussian filter to smooth item min(R,G,B)

3. Calculate the minimum value min and the maximum value max of $\min (\mathrm{R}, \mathrm{G}, \mathrm{B})$ image's pixels, set pixel value interval length variable $\boldsymbol{k}$ and moving step length variable step, and set original interval as $[n, n+k]$, in which $n=\min$. Initialize matrix summatrix and countmatrix.

4. Extract corresponding pixels in hazy image according to the value range of interval

5. Process pixels extracted in step 4 by gray stretch method, and add the result into matrix summatrix in corresponding pixels, add 1 into matrix countmatrix in corresponding pixels.

6. If item $n+k+$ step is smaller than item max, execute expression $n=n+$ step and return to step 4 . If it is the first time item $n+k+$ step bigger than item max, and change the interval into [n+step, max], return to step 4. Otherwise we execute the next step.

7. Execute $g(x, y)=\operatorname{summatrix}(x, y) / \operatorname{countmatrix}(x, y)$, and we can get final result $g(x, y)$.

Figure 2 shows the flow chart of the algorithm:

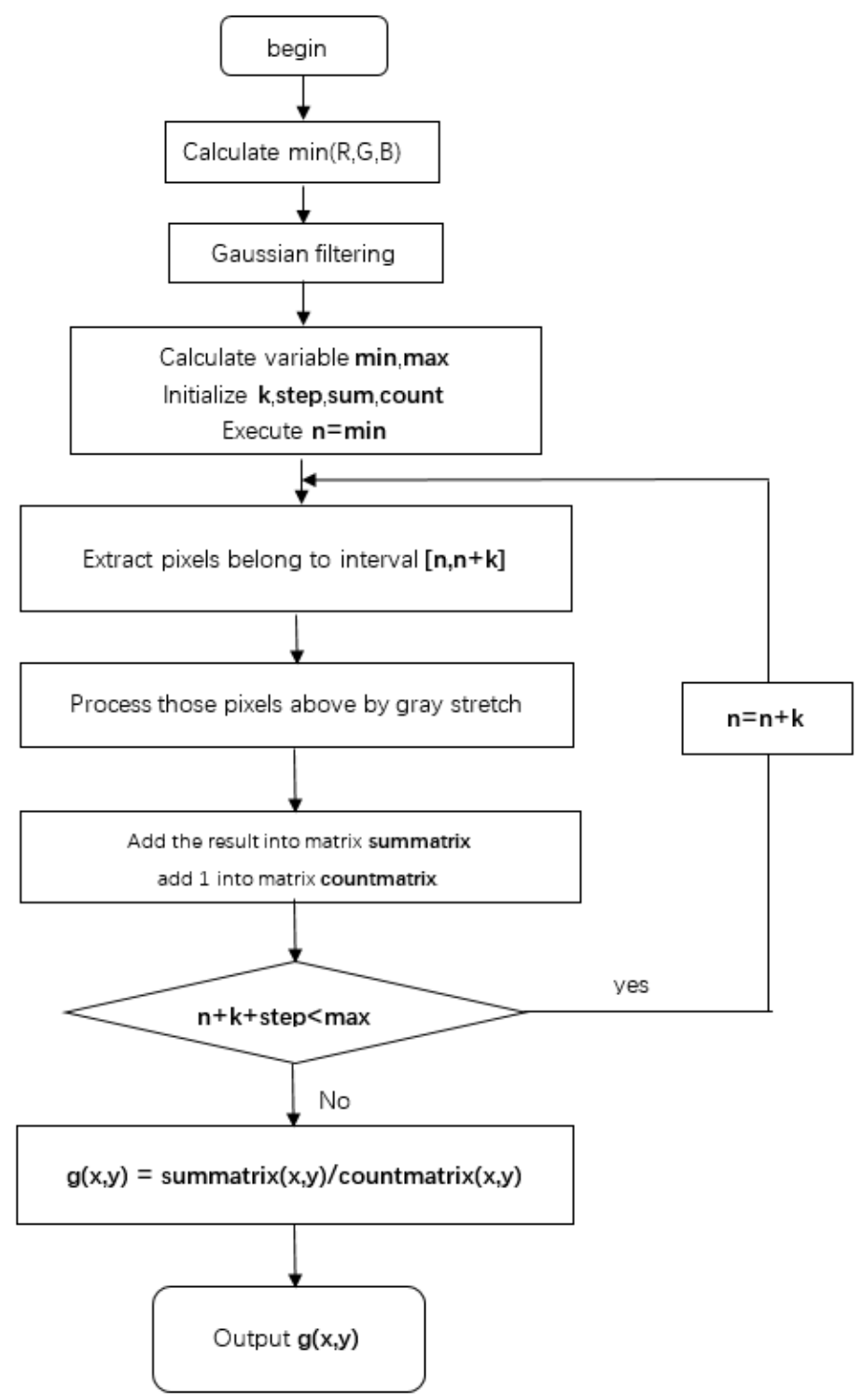

Figure 2 The flow chart of the algorithm 

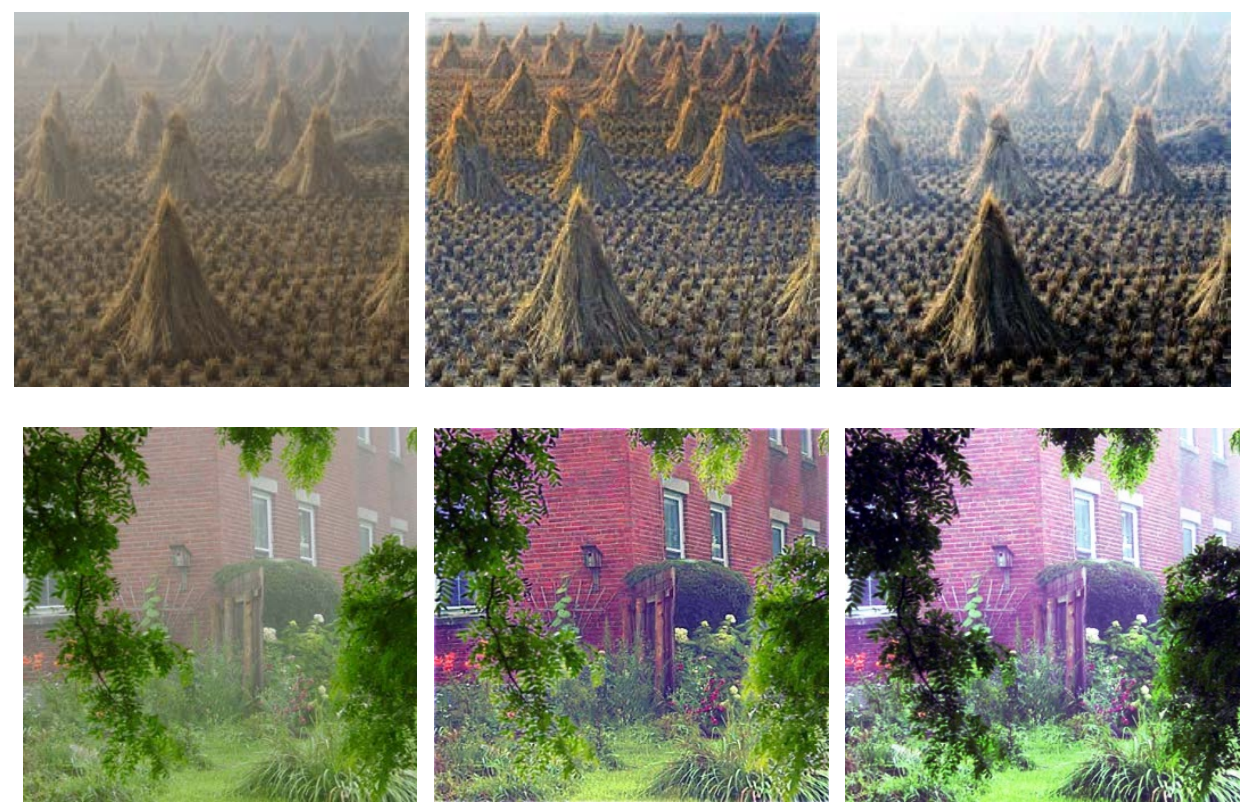

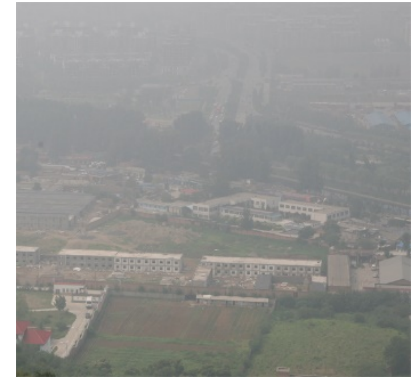

(a)

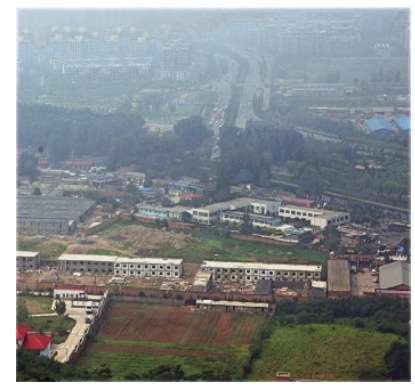

(c)

Figure 3 experimental results (a)Original image (b) Our method (c)processed by histogram equalization

Figure 3 shows the results of our method. We can see that compared with histogram equalization method, our method takes advantage in plentiful details and much clearer in far field's scene. What's more, we can see that the results processed by histogram equalization have serious color distortion, but our method can achieve a higher color fidelity result.

But our method are too slow, because we have to ergodic min(R,G,B) image each time we need to extract pixels. So we can set all intervals in advance as follows: [ $\mathbf{m i n}, \mathbf{m i n}+\mathbf{k}],[\mathbf{m i n}+\mathbf{s}, \mathbf{m i n}+\mathbf{s}+\mathbf{k}] \ldots$ $[\min +\mathbf{n} * \mathbf{s}, \min +\mathbf{n} * \mathbf{s}+\mathbf{k}] \ldots[\min +\mathbf{n m a x} * \mathbf{s}, \max ]$.

So we only need ergodic min(R,G,B) image once. we find corresponding interval according to each pixel value, and put corresponding pixel in hazy image into interval. Then process each image by gray stretch, it can achieve the same result as above, but it is much faster than method above.

\section{Conclusion}

In this paper, we proposed an image dehazing method based on scene segmentation. Our experiments are performed and achieve high color fidelity results. What's more, our results take advantage in plentiful details and much clearer in the far field's scene. But our method still has color fidelity in some field, so we will focus on these problems to improve this method's applicability.

\section{Acknowledgement}

This work was supported by the Key technologies R\&D Program of Guangdong Province(2014B010119002), the Natural Science Foundation of Guangdong Province (Nos.2015A030310278,2016A030313473), and partly by Zhejiang Key Discipline of Instrument Science and technology(No. JL150510). 


\section{References:}

[1]. J. H. Kim, J. Y. Sim, and C. S. Kim, Single Image Dehazing Based on Contrast Enhancement, ICASSP, 1273-1276 (2011).

[2]. Land E H, Mccann J J. Lightness and retinex theory , Journel of Optical Society of America,1971,61(1971),No.1,p.1-11.

[3]. R. Tan, Visibility in Bad Weather from a Single Image Proc. IEEE Conf. Computer Vision and Pattern Recognition, June 2008.

[4]. R. Fattal, Single image dehazing, ACM Transactions on Graphics, 27 (2008), No.3,p.1-9.

[5]. Jean-Philippe T,Nicolas H. Fast visibility restoration from a single color or gray level image//Proceeding of IEEE 12th International Conference on Computer Vision New York, USA;IEEE,2009;2201-2208.

[6]. K. M. He, J. Sun, and X. O. Tang, Single image haze removal using dark channel prior, IEEE Trans. Pattern Anal. Mach. Intell. 33(2010),No.12, p.2341-2353.

[7]. S. Y. Tao, H. J. Feng, Z. H. Xu, Q. Li, Image degradation and recovery based on multiple scattering in remote sensing and bad weather condition, Optics Express 20(2012),No.15,p.16584-16595.

[8]. S. Fang, X. Xia, X. Huo, and C. Chen, Image dehazing using polarization effects of objects and airlight,Opt. Express 22(2014),No.,p. 19523-19537.

[9]. F. Liu, L. Cao, X. Shao, P. Han, and X. Bin, Polarimetric dehazing utilizing spatial frequency segregation of images,Appl. Opt. 54(2015),No.27,p. 8116-8122.

[10]. J. Mudge and M. Virgen, Real time polarimetric dehazing, Appl. Opt. 52(2013),No.9,p. 1932-1938.

[11]. C. Yeh, L. Kang, M. Lee, and C. Lin, Haze effect removal from image via haze density estimation in optical model, Opt. Express 21 (2013),No.22,p. 27127-27141.

[12]. Narasimhan S G, Nayar S K. Shedding light on the weather, Computer Vision and Pattern Recognition, 1(2003),No.1,p. 665-672.

[13]. Narasimhan S G, Nayar S K. Vision and the atmosphere, International Journal of Computer Vision, 48 (2002), No.3,p. 233-54. 